\title{
THE KLEIN BOTTLE AND MULTICOMMODITY FLOWS
}

\author{
A. SCHRIJVER \\ Received July 23, 1988 \\ Revised November 28, 1988
}

Let $G$ be an eulerian graph embedded on the Klein bottle. Then the maximum number of pairwise edge-disjoint orientation-reversing circuits in $G$ is equal to the minimum number of edges intersecting all orientation-reversing circuits. This generalizes a theorem of Lins for the projective plane. As consequences we derive results on disjoint paths in planar graphs, including theorems of Okamura and of Okamura and Seymour.

\section{Introduction}

In [5] we proved:

Theorem 1. Let $G=(V, E)$ be a planar bipartite graph embedded in the plane. Let $I_{1}$ and $I_{2}$ be two of its faces. Then there exist pairwise edge-disjoint cuts $\delta\left(X_{1}\right), \ldots, \delta\left(X_{t}\right)$ so that for each two vertices $v, w$ with $v, w \in b d\left(I_{1}\right)$ or $v, w \in b d\left(I_{2}\right)$, the distance in $G$ from $v$ to $w$ is equal to the number of cuts $\delta\left(X_{j}\right)$ separating $v$ and $w$.

For $X \leqq V, \delta(X)$ denotes the set of edges with exactly one of its end points in $X$. Cut $\delta(X)$ is said to separate $v$ and $w$ if $v \neq w$ and $|\{v, w\} \cap X|=1$. We denote the boundary of $I$ by $b d(I)$. Faces are considered as open regions.

In this paper we derive from Theorem 1 some new results on graphs embedded on the Klein bottle and on plane multicommodity flows, and some known results due to Okamura, Okamura and Seymour, and Lins.

\section{Graphs on the Klein bottle}

Let $G=(V, E)$ be a graph embedded on the Klein bottle. We can represent the Klein bottle as obtained from the 2 -sphere by adding two cross-caps. A circuit $C$ in $G$ is called orientation-preserving if after one turn of $C$ the meaning of 'left' and 'right' is unchanged. It is called orientation-reversing if after one turn of $C$ the meaning of 'left' and 'right' is exchanged.

Thus a circuit is orientation-preserving if and only if it passes the cross-caps an even number of times. It is orientation-reversing if and only if it passes the cross-caps an odd number of times. Hence the orientation-reversing circuits form a "binary clut-

AMS subject classification (1980): 05C10, 98CXX. 
ter" in the sense of Seymour [6]: if $C_{1}, C_{2}, C_{3}$ are (the edge sets of) orientation-reversing circuits, then the symmetric difference $C_{1} \Delta C_{2} \Delta C_{3}$ contains an orientationreversing circuit.

This implies that the inclusion-wise minimal edge sets intersecting all orientation-reversing circuits are exactly the inclusion-wise minimal sets in

$$
\{D \subseteq E|| D \triangle C \mid \text { is odd for each orientation-reversing circuit } C\} \text {. }
$$

In fact, it follows from our results that the hypergraph of orientation-reversing circuits, as well as its blocker (1), have the weak MFMC-property (Seymour [6]).

\section{The minimum length of an orientation-reversing circuit}

We first derive from Theorem 1:

Theorem 2. Let $G=(V, E)$ be a bipartite graph embedded on the Klein bottle. Then the minimum length of any orientation-reversing circuit in $G$ is equal to the maximum number of pairwise disjoint edge sets each intersecting all orientation-reversing circuits.

Proof. Clearly, the maximum is not larger than the minimum. To show equality, we may assume that each face of $G$ is orientable, i.e., contains no cross-cap. Indeed, if a face contains a cross-cap, we can add to $G$ a path over this cross-cap, in such a way that the graph remains bipartite and such that the minimum-length of an orientationreversing circuit remains unchanged (by taking the path long enough).

Let $C_{1}$ be a minimum-length orientation-reversing circuit in $G$, say with length $t_{1}$. We 'cut open' the Klein bottle $S$ along $C_{1}$. In this way we obtain a bordered surface $S^{\prime}$, with a 1 -sphere $C_{1}^{\prime}$ as border, so that $S$ arises from $S^{\prime}$ by identifying opposite points on $C_{1}^{\prime}$. So $S^{\prime}$ is a Möbius strip. Let $i: S^{\prime} \rightarrow S$ be the identification map. The graph $G^{\prime}:=i^{-1}[G]$ is a graph on $S^{\prime}$, where $C_{1}^{\prime}=i^{-1}\left[C_{3}\right]$.

As each face of $G$ is orientable, also each face of $G^{\prime}$ (in $S^{\prime}$ ) is orientable. Therefore, $G^{\prime}$ contains an orientation-reversing circuit (in $S^{\prime}$ ). Let $C_{2}$ be a minimum-length orientation-reversing circuit in $G^{\prime}$, say with length $t_{2}$. We may assume that $C_{2}$ is edge-disjoint from $C_{J}$ (by adding parallel edges). Next we 'cut open' the Möbius strip $S^{\prime}$ along $C_{2}$. We now obtain a cylinder $S^{\prime \prime}$, with boundary two 1 -spheres $B_{1}$ and $B_{2}$. (It is a deformed cylinder if $B_{1}$ and $B_{2}$ have points in common.) The Klein bottle $S$ arises from $S^{\prime \prime}$ by identifying opposite points on $B_{1}$ and by identifying opposite points on $B_{2}$. Let $i^{\prime}: S^{\prime \prime} \rightarrow S$ be the identification map, and let $G^{\prime \prime}:=\left(i^{\prime}\right)^{-1}[G]$. So $G^{\prime \prime}$ is a planar graph, embeddable in the plane $\mathbf{R}^{2}$, so that two of its faces $I_{1}(=$ unbounded face) and $I_{2}$ have the following properties:

(2) (i) the boundary of $I_{1}$ is a circuit $D_{1}$ of length $2 t_{1}$, and the boundary of $I_{2}$ is a circuit $D_{2}$ of length $2 t_{2}$;

(ii) $S$ arises from $\mathbf{R}^{2} \backslash\left(I_{1} \cup I_{2}\right)$ by identifying pairs of opposite points on $D_{1}$ and by identifying pairs of opposite points on $D_{2}$.

We may assume that $S^{\prime \prime}=\mathbf{R}^{2} \backslash\left(I_{1} \cup I_{2}\right)$.

Since $t_{1}$ is the minimum length of an orientation-reversing circuit in $G$, each pair of opposite vertices on $D_{1}$ has distance exactly $t_{1}$. Since $t_{2}$ is the minimum length of an orientation-reversing circuit in $G$, each pair of opposite vertices on $D_{2}$ has distance exactly $t_{2}$. 
By Theorem 1, there exist pairwise disjoint cuts $\delta\left(X_{1}\right), \ldots, \delta\left(X_{t}\right)$ so that for each two vertices $v$ and $w$ of $G^{\prime \prime}$ with $v, w \in b d\left(I_{1}\right)$ or $v, w \in b d\left(I_{2}\right)$, the distance in $G^{\prime \prime}$ from $v$ to $w$ is equal to the number of cuts $\delta\left(X_{j}\right)$ separating $v$ and $w$. We may assume that each $\delta\left(X_{j}\right)$ separates at least one such pair $v, w$ (all other cuts can be deleted), and that each $\delta\left(X_{j}\right)$ is a minimal nonempty cut (inclusion-wise).

Each cut $\delta\left(X_{j}\right)$ intersects any subpath $P$ of $D_{1}$ of length $t_{1}$ at most once (as $P$ is intersected by $t_{1}$ of the $\delta\left(X_{j}\right)$, as $P$ is a shortest path between its two end points). So if $\delta\left(X_{j}\right)$ intersects $D_{1}$, it intersects $D_{1}$ exactly twice, in two opposite edges. Simitarly, if $\delta\left(X_{j}\right)$ intersects $D_{2}$, it intersects $D_{2}$ exactly twice, in two opposite edges.

We can classify the $\delta\left(X_{j}\right)$ into three classes:

(3) (i) those intersecting both $D_{1}$ and $D_{2}$, say $\delta\left(X_{1}\right), \ldots, \delta\left(X_{s}\right)$;

(ii) those intersecting $D_{1}$ but not $D_{2}$, say $\delta\left(X_{s+1}\right), \ldots, \delta\left(X_{t_{1}}\right)$;

(iii) those intersecting $D_{2}$ but not $D_{1}$, say $\delta\left(X_{t_{1}+1}\right), \ldots, \delta\left(X_{t}\right)$.

Note that $t_{2}=s+\left(t-t_{1}\right)$, and hence $s=t_{1}+t_{2}-t$.

First consider $\delta\left(X_{1}\right), \ldots, \delta\left(X_{s}\right)$. Each such $\delta\left(X_{j}\right)$ is (since it is a minimal cut) the set of edges of $G^{\prime \prime}$ intersected by two curves $\Gamma_{1}$ and $\Gamma_{2}$, where $\Gamma_{1}$ connects points $p^{\prime}$ on $D_{1}$ and $p^{\prime \prime}$ on $D_{2}$, while $\Gamma_{2}$ connects points $q^{\prime}$ on $D_{1}$ and $q^{\prime \prime}$ on $D_{2}$, in such a way that $p^{\prime}$ and $q^{\prime}$ are opposite on $D_{1}$, and $p^{\prime \prime}$ and $q^{\prime \prime}$ are opposite on $D_{2}$ :

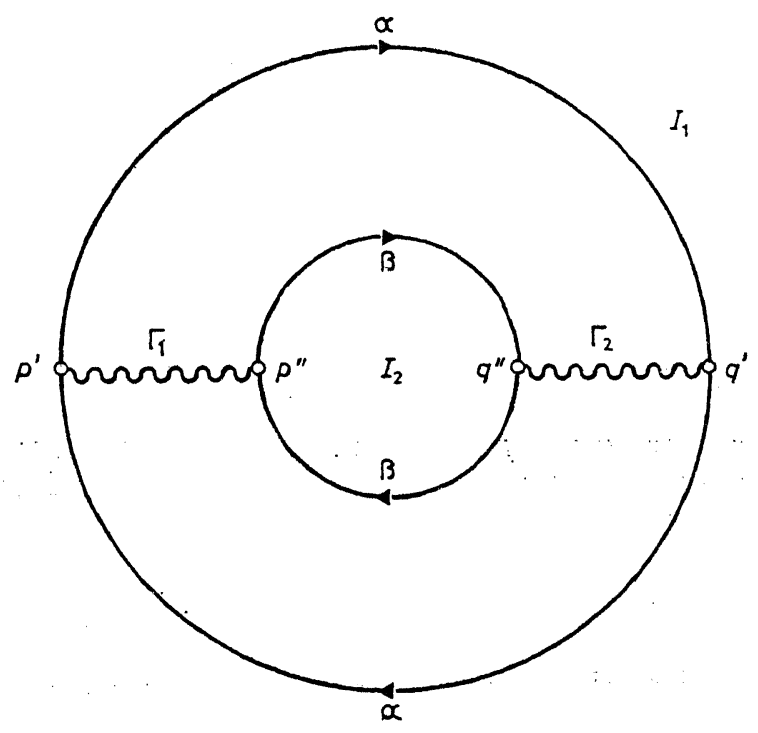

Fig. 1

The space $i^{\prime}\left[S^{\prime \prime} \backslash\left(\Gamma_{1} \cup \Gamma_{2}\right)\right]$ is orientable, since it arises from Fig. 1 by identifying the two curves $\alpha$ (in the orientation given), and similarly the two curves $\beta$, which yields a cylinder. Hence $i^{\prime}\left[\Gamma_{1} \cup \Gamma_{2}\right]$ intersects all orientation-reversing closed curves on $S$, and hence $i^{\prime}\left[\delta\left(X_{j}\right)\right]$ is a set of edges in $G$ intersecting all orientation-reversing circuits. 
Similarly, each set

$$
i^{\prime}\left[\delta\left(X_{s+j}\right) \cup \delta\left(X_{t_{1}+j}\right)\right],
$$

for $j=1, \ldots, t_{1}-s$, intersects all orientation-reversing circuits in $G$ (note $t_{1}+\left(t_{1}-s\right) \leqq$ $\leqq t_{2}+t_{1}-s=t$ as $\left.t_{1} \leqq t_{2}\right)$. Now $\delta\left(X_{s+j}\right)$ is the set of edges intersected by a curve $\Gamma_{1}$ connecting two opposite points $p^{\prime}$ and $q^{\prime}$ on $D_{1}$, while $\delta\left(X_{t_{1}+j}\right)$ is the set of edges intersected by a curve $\Gamma_{2}$ connecting two opposite points $p^{\prime \prime}$ and $q^{\prime \prime}$ on $D_{2}$ :

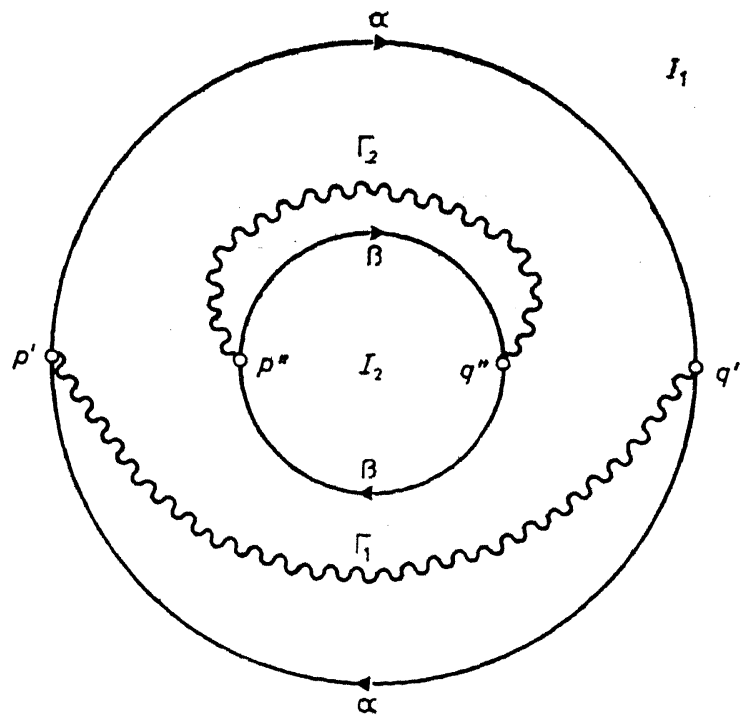

Fig. 2

Again the space $i^{\prime}\left[S^{\prime \prime} \backslash\left(\Gamma_{1} \cup \Gamma_{2}\right)\right]$ is orientable, since it arises from Fig. 2 by identifying the two curves $\alpha$ and the two curves $\beta$, yielding again a cylinder. So $i^{\prime}\left[\Gamma_{1} \cup \Gamma_{2}\right]$ intersects all orientation-reversing closed curves in $S$, and hence (4) intersects all orientation-reversing closed curves in $S$, and hence (4) intersects all orientation-reversing circuits in $G$.

Combining,

$$
i^{\prime}\left[\delta\left(X_{1}\right)\right], \ldots, i^{\prime}\left[\delta\left(X_{s}\right)\right], i^{\prime}\left[\delta\left(X_{s+1}\right) \cup \delta\left(X_{t_{1}+1}\right)\right], \ldots, i^{\prime}\left[\delta\left(X_{t_{1}}\right) \cup \delta\left(X_{2 t_{1}-s}\right)\right]
$$

are $t_{1}$ pairwise edge-disjoint sets of edges of $G$, each intersecting all orientation-reversing circuits.

Note. In fact, the proof shows that it suffices to require that each nullhomotopic circuit in $G$ is even (instead of $G$ being bipartite). Indeed, this implies that the graph $G^{\prime \prime}$ described in the proof above is bipartite. 


\section{The max-flow min-cut property}

Theorem 2 implies the following. Let $G=(V, E)$ be a graph embedded on the Klein bottle. Let

(6) $\mathscr{C}:=$ collection of orientation-reversing circuits in $G$;

$b(\mathscr{C}):=$ collection of edge-sets intersecting each orientation-reversing circuit in $G$.

Then the hypergraph $(E, b(\mathscr{C}))$ has the weak MFMC-property, in the sense of Seymour [6]. That is, the vertices of the polytope in $\mathbf{R}^{E}$ determined by:

$$
\begin{aligned}
& \text { (i) } 0 \leqq x(e) \leqq 1 \quad(e \in E) \text {, } \\
& \text { (ii) } \sum_{e \in D} x(e) \geqq 1 \quad(D \in b(\mathscr{C})) \text {, }
\end{aligned}
$$

are $\{0,1\}$-vectors. These vectors are exactly the characteristic vectors of subsets of $E$ containing an orientation-reversing circuit.

This follows from the fact that, for any $l: E \rightarrow \mathbf{Z}_{+} \backslash\{0\}$, the minimum value of

$$
\sum_{e \in E} l(e) x(e)
$$

over (7) is achieved by an integer vector $x$. To see this, we may assume that $l(e)$ is even for each $e \in E$. Now replace each edge $e$ of $G$ by a path of length $l(e)$. We obtain a bipartite graph $G^{\prime}$. Let $C^{\prime}$ be a minimum-length orientation-reversing circuit in $G^{\prime}$. By Theorem 2 there exist pairwise disjoint edge sets $D_{1}^{\prime}, \ldots, D_{i}^{\prime}$ in $G^{\prime}$ each intersecting all orientation-reversing circuits in $G^{\prime}$, so that $t$ is equal to the number of edges in $C^{\prime}$. Let $C, D_{1}, \ldots, D_{t}$ be the 'projections' of $C^{\prime}, D_{1}^{\prime}, \ldots, D_{t}^{\prime}$ to $G$. Then

$$
t=\sum_{e \in E} l(e) \chi^{c}(e)
$$

where $\chi^{c}$ denotes the characteristic vector of $C$. Since $D_{1}, \ldots, D_{t}$ give a dual solution to (7) of value $t$, it follows that $\chi^{c}$ is an optimum solution.

By Lehman's theorem [1] the weak MFMC-property is maintained under taking blocking hypergraphs. So also $\mathscr{C}$ has the weak MFMC-property. That is, the vertices of the polytope in $\mathbf{R}^{E}$ determined by:

$$
\begin{array}{ll}
\text { (i) } \quad 0 \leqq x(e) \leqq 1 & (e \in E), \\
\text { (ii) } \sum_{e \in C} x(e) \geqq 1 & (C \in \mathscr{C}),
\end{array}
$$

are $\{0,1\}$-vectors. These vectors are exactly the characteristic vectors of sets in $b(\mathscr{C})$. In the following section we show that a stronger property holds. 


\section{Packing orientation-reversing circuits}

We derive from the previous results:

Theorem 3. Let $G=(V, E)$ be an eulerian graph embedded on the Klein bottle. Then the maximum number of pairwise edge-disjoint orientation-reversing circuits is equal to the minimum number or edges intersecting all orientation-reversing circuits.

Proof. Clearly, the maximum is not more than the minimum. Suppose equality does not hold, and let $G$ form a counterexample with

$$
\sum_{v \in V} 2^{\operatorname{deg}(v)}
$$

as small as possible (where $\operatorname{deg}(v)$ denotes the degree of $v$ ). Let $D$ be a set of edges intersecting all orientation-reversing circuits in $G$, of minimum size $t=|D|$. Since $t$ is equal to the minimum value of

$$
\sum_{e \in E} x(e)
$$

over (10) (as (10) is the convex hull of the characteristic vectors of edge-sets intersecting all orientation-reversing circuits), there exist, by linear programming duality, orientation-reversing circuits $C_{1}, \ldots, C_{k}$ (pairwise different) and reals $\lambda_{1}, \ldots, \lambda_{k}>0$, so that:

$$
\begin{aligned}
& \text { (i) } \sum_{i=1}^{k} \lambda_{i}=t \\
& \text { (ii) } \sum_{i=1}^{k} \lambda_{i} \chi^{C_{i}(e) \leqq 1 \quad(e \in E) .}
\end{aligned}
$$

In fact, what we must show is that each $\lambda_{i}$ can be taken to be 1 .

Consider a vertex $v$ of $G$, and the edges $e_{1}, \ldots, e_{2 d}$ incident to $v$, in cyclic order:

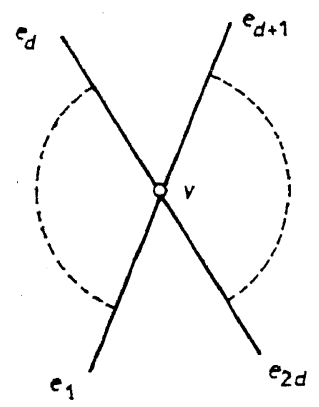

Fig. 3

Thus $e_{1}$ and $e_{d+1}$ are 'opposite', and similarly $e_{2}$ and $e_{d+2}, e_{3}$ and $e_{d+3}, \ldots, e_{d}$ and $e_{2 d}$. We show that for each circuit $C_{i}$ and each $j=1, \ldots, d$ :

$$
C_{i} \text { passes } e_{j} \Leftrightarrow C_{i} \text { passes } e_{d+j} \text {. }
$$


Having shown this for each vertex $v$, each $j$ and each $C_{i}$, it follows that the $C_{1}, \ldots, C_{k}$ are pairwise edge-disjoint. Since $k \geqq \mathrm{t}$ (as $\lambda_{i} \leqq 1$ for all $i$ ), this proves the theorem. Suppose (14) does not hold for some $v, i, j$. Without loss of generality, $i=1$, $j=1$, and $C_{1}$ passes $e_{1}$ and $e_{m}$ for some $m$ with $2 \leqq m \leqq d$. Now replace Fig. 3. by:

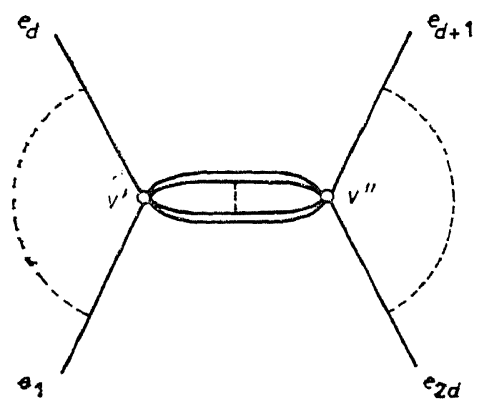

Fig. 4

where there are $d-2$ parallel edges connecting the new vertices $v^{\prime}$ and $v^{\prime \prime}$. Let $G^{\prime}$ be the new graph obtained. So $G$ arises from $G^{\prime}$ by contracting the parallel edges connecting $v^{\prime}$ and $v^{\prime \prime}$. (If $d=2$, we identify $v^{\prime}$ and $v^{\prime \prime}$.) Graph $G^{\prime}$ is eulerian again, with sum (11) smaller than for $G$. So by the minimality hypothesis, the theorem to be proved holds for $G^{\prime}$.

Let $D^{\prime}$ be a minimum-sized set of edges in $G^{\prime}$ intersecting all orientation-reversing circuits in $G^{\prime}$. Let $t^{\prime}:=\left|D^{\prime}\right|$. If $t^{\prime} \geqq t, G^{\prime}$ would contain $t$ pairwise edge-disjoint orientation-reversing circuits. After identifying $v^{\prime}$ and $v^{\prime \prime}$, this gives $t$ pairwise edgedisjoint orientation-reversing circuits in $G$, contradicting our assumption. So $t^{\prime}<t$.

We show $t^{\prime} \leqq t-2$. Let $\bar{D}$ be the set of edges in $G^{\prime}$ corresponding to $D$. By the minimality of $D, D$ intersects each orientation-reversing circuit in $G$ an odd number of times, and each orientation-preserving circuit in $G$ an even number of times. Hence also $\bar{D}$ intersects each orientation-reversing circuit in $G^{\prime}$ an odd number of times, and each orientation-preserving circuit in $G^{\prime}$ an even number of times. By the minimality of $D^{\prime}$, also $D^{\prime}$ has odd intersection with each orientation-reversing circuit, and even intersection with each orientation-preserving circuit in $G^{\prime}$. This implies that the symmetric difference $\bar{D} \triangle D^{\prime}$ has even intersection with each circuit in $G^{\prime}$. So $\bar{D} \triangle D^{\prime}$ is a cut in $G^{\prime}$, and hence, as $G^{\prime}$ is eulerian, $\left|\bar{D} \Delta D^{\prime}\right|$ is even. That is, $|\bar{D}| \equiv\left|D^{\prime}\right|(\bmod 2)$. Therefore, as $t^{\prime}<t$, we know $t^{\prime} \leqq t-2$.

Let $\pi$ denote the set of parallel edges in $G^{\prime}$ connecting $v^{\prime}$ and $v^{\prime \prime}$. We show that $\pi \subseteq D^{\prime}$. If not, $\pi \neq \emptyset$, and hence $d \geqq 3$. Let $e \in \pi \backslash D^{\prime}$. Then $D^{\prime} \backslash \pi$ intersects all orientation-reversing circuits in $G^{\prime}$, and hence (after contracting the edges in $\pi$ ) also all orientation-reversing circuits in $G$. However, $\left|D^{\prime} \backslash \pi\right| \leqq\left|D^{\prime}\right|<|D|$, contradicting the minimality of $D$.

Let

$$
D^{\prime \prime}:=\left(D^{\prime} \backslash \pi\right) \cup\left\{e_{1}, \ldots, e_{d}\right\}
$$

Since $|\pi|=d-2$, we know $\left|D^{\prime \prime}\right| \leqq t^{\prime}+2 \leqq t$. Let $\overline{D^{\prime \prime}}$ be the set of edges in $G$ corresponding to $D^{\prime \prime}$. Then $\overline{D^{\prime \prime}}$ intersects all orientation-reversing circuits in $G$ (since each 
orientation-reversing circuit in $G$ intersects $\left\{e_{1}, \ldots, e_{d}\right\}$ or comes from an orientationreversing circuit in $G^{\prime}$ not intersecting $\pi$ ). So $\left|\overline{D^{\prime \prime}}\right|=t$. Hence $\chi^{\overline{D^{\prime \prime}}}$ attains the minimum of (12) over (10). So by complementary slackness, $\left|\overline{D^{\prime \prime}} \cap C_{1}\right|=1$. This contradicts the fact that $e_{1}, e_{m} \in \overline{D^{\prime \prime}} \cap C_{1}$.

Theorem 3 generalizes a theorem of Lins [2], which in fact is Theorem 3 with respect to the projective plane instead of the Klein bottle. If $G$ is a graph embedded on the projective plane, we can insert a cross-cap in one of the faces of $G$. This transforms the projective plane to a Klein bottle. As the meaning of 'orientation-reversing' is not changed by this insertion (for the circuits in $G$ ), it reduces Lins' theorem to Theorem 3.

Theorem 3 cannot be extended to compact surfaces with more than two crosscaps, as we can embed $K_{5}$ on such a surface in such a way that the orientation-reversing circuits are exactly the odd-sized circuits. Then the maximum number of pairwise edge-disjoint orientation-reversing circuits is equal to 2, while not less than 4 edges are necessary to intersect all orientation-reversing circuits.

\section{Plane multicommodity flows}

From Theorem 3 we derive a new result on the existence of pairwise edgedisjoint paths in a planar graph. Let $G=(V, E)$ be a graph, and let $r_{1}, \ldots, r_{k}$, $s_{1}, \ldots, s_{k}$ be vertices of $G$. We consider the following two conditions:

$$
\begin{gathered}
\text { (parity condition): for each vertex } v \text { of } G: \\
\operatorname{deg}(v)+\left|\left\{i \in\{1, \ldots, k\} \mid r_{i}=v\right\}\right|+\left|\left\{i \in\{1, \ldots, k\} \mid s_{i}=v\right\}\right| \text { is even; } \\
\text { (cut condition): for each } X \subseteq V: \\
|\delta(X)| \geqq \text { number of pairs } r_{i}, s_{i} \text { separated by } \delta(X) .
\end{gathered}
$$

Theorem 4. Let $G=(V, E)$ be a planar graph embedded in the plane $\mathbf{R}^{2}$. Let $r_{1}, \ldots$ $\ldots, r_{k}, s_{1}, \ldots, s_{k}$ be vertices of $G$ satisfying the parity condition. Let $r_{1}, \ldots, r_{k}$ be incident to the unbounded face $I_{1}$ in clockwise order. Let $s_{1}, \ldots, s_{k}$ be incident to some other face $I_{2}$ in anti-clockwise order. Then there exist pairwise edge-disjoint paths $P_{1}, \ldots, P_{k}$ where $P_{i}$ connects $r_{i}$ and $s_{i}(i=1, \ldots, k)$, if and only if the cut condition is satisfied.

Proof. Since the cut condition trivially is a necessary condition, we only show sufficiency. Let the cut condition be satisfied. We can extend $\mathbf{R}^{2} \backslash\left(I_{1} \cup I_{2}\right)$ to the Klein bottle, by adding a cylinder between the boundaries of $I_{1}$ and $I_{2}$. We can extend $G$ to a graph $G^{\prime}$ on the Klein bottle adding edges $e_{1}, \ldots, e_{k}$ over this cylinder, so that $e_{i}$ connects $r_{i}$ and $s_{i}(i=1, \ldots, k)$. Then a circuit in $G^{\prime}$ is orientation-reversing if and only if it contains an odd number of edges from $e_{1}, \ldots, e_{k}$. So it suffices to show that $G^{\prime}$ contains $k$ pairwise edge-disjoint orientation-reversing circuits.

By the parity condition, $G^{\prime}$ is eulerian. So we can apply Theorem 3 . Hence it suffices to show that each set $D$ of edges of $G^{\prime}$ intersecting all orientation-reversing circuit has size at least $k$. We may assume that $D$ is a minimal set of edges in $G^{\prime}$ intersecting all orientation-reversing circuits in $G^{\prime}$. Hence $|D \cap C|$ is even for each circuit $C$ in $G$. Therefore, $D \cap E$ is a cut $\delta(X)$ in $G$. Now we have for each $i=1, \ldots, k$ :

$$
\delta(X) \text { does not separate } r_{i} \text { and } s_{i} \Rightarrow e_{i} \in D \text {. }
$$


Indeed, if $\delta(X)$ does not separate $r_{i}$ and $s_{i}$, then there exists a path $P$ in $G$ connecting $r_{i}$ and $s_{i}$ and containing an even number of edges in $D$. Now as $P \cup\left\{e_{i}\right\}$ is an orientation-reversing circuit, it intersects $D$ an odd number of times, and hence $e_{i} \in D$. Assertion (17) implies that $\left|D \cap\left\{e_{1}, \ldots, e_{k}\right\}\right|$ is not less than the number of pairs $r_{i}, s_{i}$ not separated by $\delta(X)$. Hence

$$
|D|=|D \cap E|+\left|D \cap\left\{e_{1}, \ldots, e_{k}\right\}\right| \geqq|\delta(X)|+\text { number of pairs } r_{i}, s_{i} \text { not }
$$

separated by $\delta(X) \geqq k$,

by the cut condition.

\section{A theorem of Okamura}

One can also derive a theorem of Okamura [3]:

Theorem 5. Let $G=(V, E)$ be a planar graph embedded in the plane $\mathbf{R}^{2}$. Let $I_{1}$ and $I_{2}$ be two of its faces, and let $r_{1}, \ldots, r_{k}, s_{1}, \ldots, s_{k}$ be vertices satisfying the parity condition, so that for each $i=1, \ldots, k: r_{i}, s_{i} \in b d\left(I_{1}\right)$ or $r_{i}, s_{i} \in b d\left(I_{2}\right)$. Then there exist pairwise edge-disjoint paths $P_{1}, \ldots, P_{k}$ where $P_{i}$ connects $r_{i}$ and $s_{i}(i=1, \ldots, k)$, if and only if the cut condition is satisfied.

Proof. Again, it suffices to show sufficiency. Without loss of generality, $I_{1}$ is the unbounded face, and $r_{1}, \ldots, r_{t}, s_{1}, \ldots, s_{t} \in b d\left(I_{1}\right)$ and $r_{t+1}, \ldots, r_{k}, s_{t+1}, \ldots, s_{k} \in b d\left(I_{2}\right)$. By an argument due to $\mathrm{S}$. Lins, we may assume that $r_{1}, \ldots, r_{t}, s_{1}, \ldots, s_{t}$ occur in cyclic order around $I_{1}$. To see this, first note that we may assume that the vertices $r_{1}, \ldots, r_{k}, s_{1}, \ldots, s_{k}$ are distinct and have degree 1 (as we can add a new vertex of degree 1 to any $r_{i}$ or $s_{i}$ and replace this $r_{i}$ or $s_{i}$ by the new vertex). Call two pairs $r_{i}$, $s_{i}$ and $r_{i}, s_{j}$ on $b d\left(I_{1}\right)$ crossing if $i \neq j$ and $r_{i}, r_{j}, s_{i}, s_{j}$ occur in this cyclic order around the boundary of $I_{1}$, clockwise or anti-clockwise. Suppose not all pairs of pairs $r_{i}, s_{i}$ are crossing. Then there exist $i, j$ so that $r_{i}, s_{i}$ and $r_{j}, s_{j}$ are non-crossing and so that there is no pair $r_{h}, s_{h}$ on that part of the boundary of $I_{1}$ that connects $r_{i}$ and $r_{j}$ and that does not pass $s_{i}$ and $s_{j}$. Now we can add in $I_{1}$ three new vertices $w, r_{i}^{\prime}$ and $r_{j}^{\prime}$ and four new edges as follows:

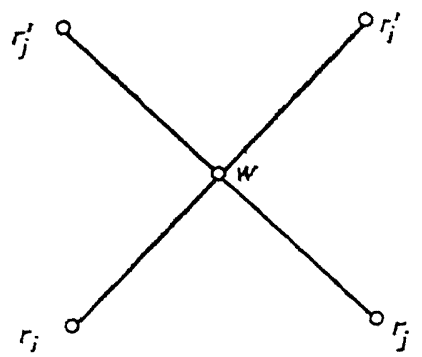

Fig. 5 
Replacing $r_{i}$ and $r_{j}$ by $r_{i}^{\prime}$ and $r_{j}^{\prime}$ does not violate the cut condition. Moreover, any pair of edge-disjoint paths $P_{i}^{\prime}, P_{j}^{\prime}$ in the extended graph, where $P_{i}^{\prime}$ connects $r_{i}^{\prime}$ and $s_{i}$ and $P_{j}^{\prime}$ connects $r_{j}^{\prime}$ and $s_{j}$, contains edge-disjoint paths $P_{i}$ and $P_{j}$, where $P_{i}$ connects $r_{i}$ and $s_{i}$ and $P_{j}$ connects $r_{j}$ and $s_{j}$.

Repeating this construction, we end up with $r_{1}, \ldots, r_{t}, s_{1}, \ldots, s_{t}$ occurring cyclically around $I_{1}$ (possibly after reordering indices and exchanging $r_{i}$ and $s_{i}$ ). Similarly, we can assume that $r_{t+1}, \ldots, r_{k}, s_{t+1}, \ldots, s_{k}$ occur cyclically around $I_{2}$. Now we can extend $\mathbf{R}^{2} \backslash\left(I_{1} \cup I_{2}\right)$ to the Klein bottle, by adding cross-caps along the boundaries of $I_{1}$ and $I_{2}$. We can extend $G$ to a graph $G^{\prime}$ on the Klein bottle by adding edges $e_{1}, \ldots, e_{k}$ over the cross-caps, so that $e_{i}$ connects $r_{i}$ and $s_{i}(i=1, \ldots$, $\ldots, k)$. Then a circuit in $G^{\prime}$ is orientation-reversing if and only if it contains an odd number of edges from $e_{1}, \ldots, e_{k}$. The remainder of the proof is exactly the same as that of Theorem 4.

Okamura's theorem has as special case the theorem of Okamura and Seymour [4], where $r_{1}, \ldots, r_{k}, s_{1}, \ldots, s_{k}$ are all on the boundary of one face.

Acknowledgement. I thank the referee for carefully reading the paper and for giving helpful suggestions.

\section{References}

[1] A. Lehman, On the width-length inequality, Mathematical Programming, 17 (1979) 403-417.

[2] S. LINS, A minimax theorem on circuits in projective graphs, Journal of Combinatorial Theory $(B)$, 30 (1981) 253-262.

[3] H. ORamura, Multicommodity flows in graphs, Discrete Applied Mathematics, 6 (1983) 55-62.

[4] H. ORAMura and P. D. SEYMOUR, Multicommodity flows in planar graphs, Journal of Combinatorial Theory (B), 31 (1981) 75-81.

[5] A. SCHRIVIER, Distances and cuts in planar graphs, Journal of Combinatorial Theory (B), 46 (1989), 46-57.

[6] P. D. Seymour, The matroids with the max-flow min-cut property, Journal of Combinatorial Theory (B), 23 (1977) 189-222.

\section{A. Schrijver}

Mathematical Centre;

Kruislaan 413;

1098 SJ Amsterdam,

The Netherlands. 